\title{
An Observer for Permanent Magnet Synchronous Motors with Currents and Voltages as only Measurements
}

\author{
F. Poulain, L. Praly and R. Ortega
}

\begin{abstract}
We propose a Kazantzis-Kravaris-Luenberger observer for permanent magnet synchronous motors. We show how the partial differential equation, involved in its design, can be solved explicitly and we suggest a recursive procedure as a solution to the inversion problem involved in its implementation. Simulation results demonstrate the interest of this observer.
\end{abstract}

\section{INTRODUCTION}

Permanent magnet synchronous motors (PMSMs) are widely used in high performance variable frequency drives due to their high efficiency, low maintenance costs and rapid dynamic response. Since rotational transducers and their associated digital or analogue circuits give extra costs and are often complex and fragile, there has been an increasing interest in industry in control schemes without rotational sensors - the so-called sensorless control. While some successful practical implementations have already been reported, many fundamental questions remain widely open. We refer the reader to [11] for a tutorial account on the topic and to [4], [9], [10] for an overview of the recent relevant references in the control literature.

Given that high-performance controllers are readily available when position is known, it seems reasonable to try to estimate position and replace in the control scheme the actual position by its estimation. Broadly speaking, there are two approaches to rotor position estimation reported in the literature. The first one extracts the information about rotor position exploiting the fact that the magnetic saliency affects the dependence of the motor inductance on the rotor position. The methods pursuing this line of research usually involve injection of an auxiliary balanced high frequency voltage signal to probe the motor electrical subsystem. Besides the obvious undesirable feature of excitation of high frequency modes - induced by the probing signal - the quality of the estimation will depend on the effective existence of rotor saliency, which is sometimes enforced modifying the rotor slots, and the availability of good models to describe this complex electromagnetic phenomenon.

F. Poulain is with DPRS/ONERA, BP 72 - 29, av. de la division Leclerc, 92322 Châtillon, France, Email: François.Poulain@ensmp. fr

L. Praly is with CAS, ParisTech, Ecoles des Mines, 35 rue Saint Honoré, 77305, Email: Fontainebleau, France Laurent.Praly@ensmp.fr

R. Ortega is with LSS Supelec, Plateau de Moulon, 91192 Gif sur Yvette, France, Email: ortegallss.supelec.fr
The second approach, which is the one that we follow in this paper, concentrates on estimation of the motor back emf and subsequent extraction of position information from this signal. Towards this end, various observers - or even open-loop integration - of the electrical and mechanical states have been proposed.The dynamic model of the PMSM is highly nonlinear and does not fit into any of the "standard forms" for which observer designs are readily available - we refer the reader to the recent book [3] for a list of references on general observer theory, and to [10] for an overview of their application in motor control. The problem is further complicated by the fact that the state becomes unobservable at zero speed.

The contribution of the paper is to show how a Kazantzis-Kravaris-Luenberger (KKL) observer [6] can be designed and implemented. The starting point for our calculations is the classical two-phase $(\alpha, \beta)$ model, expressed in a fixed frame. To this motor representation we apply a change of coordinates to render the system amenable for an explicit construction of a KKL observer. Then we propose a recursive procedure to solve the inversion problem involved in its implementation. Simulations, even in a strongly corrupted context, demonstrate the interest of the method we propose.

The paper is organized as follows. In Section II we review the KKL observers. In Section III we present the proposed observer, and then summarize in Section IV all observer equations. Section V contains some simulation results. We wrap up the paper in Section VI with some concluding remarks. The observability analysis and an extension, to the unknown parameter case, of the proposed KKL observer are reported in an extended version of this paper, which is available upon request to the authors.

\section{QUICK REVIEW ON THE \\ KAZANTZIS-KRAVARIS-LUENBERGER OBSERVERS}

The original concept of Luenberger observers [8] has been extended to non linear systems by Kazantzis and Kravaris [6]. We summarize below its non local version as it has been developed by Andrieu and Praly [2]. For a system whose dynamics admit the model :

$$
\dot{x}=f(x, t) \quad, \quad y=h(x, t)
$$

with state $x$ in $\mathbb{R}^{n}$ and measurement $y$ in $\mathbb{R}^{p}$, a KKL observer takes the form :

$$
\dot{z}_{\lambda_{j}}=\lambda_{j} z_{\lambda_{j}}+y \quad, \quad \hat{x}=T^{*}\left(z_{\lambda_{1}}, \ldots, z_{\lambda_{m}}, t\right)
$$

with state $z=\left(z_{\lambda_{1}}, \ldots, z_{\lambda_{m}}\right)$ in $\mathbb{C}^{m p}$ and output $\hat{x}$, an 
estimate of $x$, and where the $\lambda_{j}$ are complex numbers. The design of this observer consists in choosing the number $m$, the $\lambda_{j}$ and the function $T^{*}$. For this, the following two step procedure is proposed in [2] :

i) For each complex number $\lambda$, find a solution $T_{\lambda}: \mathbb{R}^{n} \times$ $\mathbb{R} \rightarrow \mathbb{C}^{p}$ to the following partial differential equation :

$$
\frac{\partial T_{\lambda}}{\partial x}(x, t) f(x, t)+\frac{\partial T_{\lambda}}{\partial t}(x, t)=\lambda T_{\lambda}(x, t)+h(x, t)
$$

Such a solution is guaranteed to exist, at least for $x$ in a compact set, provided the real part of $\lambda$ is sufficiently negative. Actually, a solution can be obtained as :

$$
T_{\lambda}(x, t)=\int_{-\infty}^{t} \exp (\lambda(t-s)) Y(x, t, s) d s
$$

where $Y(x, t, s)$ denotes the evaluation at time $s$ of the output $y$ along the solution of (1), starting from $x$ at time $t$. Also following [2, Section 2.4], if the system is completely observable uniformly in $t$, then there exists $m$ such that, by picking the $\lambda_{j}$ with a sufficiently large module, then, for all $t$, the function $x \mapsto T=\left(T_{\lambda_{1}}(x, t), \ldots, T_{\lambda_{m}}(x, t)\right)$ is uniformly injective, at least when restricted to a compact set.

ii) $T$ being injective in $x$ for all $t$, there exists a uniquely defined function $z \in T\left(\mathbb{R}^{n}, t\right) \mapsto T^{*}(z, t)$ which satisfies :

$$
T^{*}(T(x, t), t)=x .
$$

To get the function $T^{*}$ to be used in the observer, it remains to extend its definition from $T\left(\mathbb{R}^{n}, \mathbb{R}\right)$ to the entire set $\mathbb{C}^{m p} \times \mathbb{R}$. In the following, we define $T^{*}(z, t)$ simply as a best least square estimate, i.e.

$$
T^{*}(z, t) \in \operatorname{Arg} \min _{x}|z-T(x, t)|^{2}
$$

\section{OBSERVER DESIGN}

\section{A. A design model}

A simplified model for the dynamics of the PMSM is the following two-phase model (see [5, (9.1)]) :

$$
\begin{aligned}
L \dot{i_{\alpha}} & =-R_{s} i_{\alpha}+\Phi \omega \sin \theta+v_{\alpha} \\
L \dot{i_{\beta}} & =-R_{s} i_{\beta}-\Phi \omega \cos \theta+v_{\beta} \\
J \dot{\omega} & =P \Phi\left(-i_{\alpha} \sin \theta+i_{\beta} \cos \theta\right)-B \omega-\tau_{l} \\
\dot{\theta} & =\omega
\end{aligned}
$$

where $\left(i_{\alpha}, i_{\beta}\right)$ and $\left(v_{\alpha}, v_{\beta}\right)$ are mixed phase currents and voltages expressed in a fixed frame, $\omega$ is the angular velocity, $P$ is the number of pole pairs, $\tau_{l}$ is the load torque, $J$ and $B$ are the moment of inertia and the friction constant, $\Phi$ is the magnetic flux and $L$ is the inductance.

To get a model which will be useful for writing the observer, we use the state coordinates :

$$
\begin{gathered}
\mathcal{X}_{1}=\frac{P \Phi}{J} \cos \theta+\frac{L P}{J} i_{\alpha}, \quad \mathcal{X}_{2}=-\frac{P \Phi}{J} \sin \theta-\frac{L P}{J} i_{\beta} \\
\mathcal{X}_{3}=\omega, \quad \mathcal{X}_{4}=\frac{\tau_{l}}{J}, \quad \psi_{1}=i_{\alpha}, \quad \psi_{2}=i_{\beta}
\end{gathered}
$$

and the input/outputs signals :

$u_{1}=v_{\alpha}, \quad u_{2}=v_{\beta}, \quad y_{1}=i_{\alpha}, \quad y_{2}=i_{\beta}$. (4)

Note that, $i_{\alpha}$ and $i_{\beta}$ are both state components and outputs. We use two different symbols to distinguish among these roles, $\psi_{i}$ when it is a state component and $y_{i}$ when it is an output.

For the $\mathcal{X}_{i}$ components, we get :

$$
\begin{gathered}
\dot{\mathcal{X}}_{1}=\frac{P}{J}\left(-R_{s} y_{1}+u_{1}\right), \quad \dot{\mathcal{X}}_{2}=-\frac{P}{J}\left(-R_{s} y_{2}+u_{2}\right) \\
\dot{\mathcal{X}}_{3}=y_{2} \mathcal{X}_{1}+y_{1} \mathcal{X}_{2}-\frac{B}{J} \mathcal{X}_{3}-\mathcal{X}_{4}, \quad \dot{\mathcal{X}}_{4}=0
\end{gathered}
$$

where the last equation follows from the design assumption that $\tau_{l}$ is constant. These equations define a system which is linear and time varying with state $\mathcal{X}$, i.e.

$$
\dot{\mathcal{X}}=\mathcal{A}(t) \mathcal{X}+\mathcal{B}(t) \text {. }
$$

So its solution at time $s$ depends linearly on its value at time $t$, i.e. :

$$
\mathcal{X}(s)=\Phi_{\mathcal{X}}(s, t) \mathcal{X}(t)+\Psi_{\mathcal{X}}(s, t) .
$$

For the $\psi_{j}$ components, we get ${ }^{1}$ :

$$
\begin{aligned}
& \dot{\psi}_{1}=-\frac{R_{s}}{L} \psi_{1}-\mathcal{X}_{3}\left(\frac{J}{L P} \mathcal{X}_{2}+y_{2}\right)+\frac{u_{1}}{L} \\
& \dot{\psi}_{2}=-\frac{R_{s}}{L} \psi_{2}-\mathcal{X}_{3}\left(\frac{J}{L P} \mathcal{X}_{1}-y_{1}\right)+\frac{u_{2}}{L}
\end{aligned}
$$

Again, this is a linear time varying system with state $\psi$ and a second degree polynomial in the $\mathcal{X}_{i}$ as input, i.e. :

$$
\dot{\psi}=\mathcal{C}(t) \psi+\sum_{i=1} e_{i}\left(\mathcal{X}^{T} \mathcal{D}_{i} \mathcal{X}\right)+\mathcal{E}(t) \mathcal{X}+\mathcal{F}(t)
$$

with $e_{1}=\left(\begin{array}{ll}1 & 0\end{array}\right)^{T}$ and $e_{2}=\left(\begin{array}{ll}0 & 1\end{array}\right)^{T}$. So its solution at time $s$ depends linearly on its value at time $t$ and as a second degree polynomial of $\mathcal{X}(t)$, i.e. :

$$
\begin{gathered}
\psi(s)=\Phi_{\psi}(s, t) \psi(t)+\Psi_{\psi \mathcal{X}, 1}(s, t) \mathcal{X}(t)+\Psi_{\psi \mathcal{X}, 0}(s, t) \\
+\sum_{i=1}^{2} e_{i}\left(\mathcal{X}(t)^{T} \Psi_{\psi \mathcal{X}, 2 i}(s, t) \mathcal{X}(t)\right)
\end{gathered}
$$

Finally, we note that, we have the relation ${ }^{2}$ :

$$
\left(\mathcal{X}_{1}-\frac{L P}{J} \psi_{1}\right)^{2}+\left(\mathcal{X}_{2}+\frac{L P}{J} \psi_{2}\right)^{2}=\frac{P^{2} \Phi^{2}}{J^{2}}
$$

We denote by $\mathfrak{S}$ the subset of states $(\mathcal{X}, \psi)$ in $\mathbb{R}^{4} \times \mathbb{R}^{2}$ which satisfy this constraint.

The observer we propose for the system (5), (8) takes the form :

$$
\dot{z}_{\lambda_{j}}=\lambda_{j} z_{\lambda_{j}}+y, \quad \hat{x}=T^{*}\left(z_{\lambda_{1}}, \ldots, z_{\lambda_{m}}, t\right)
$$

with $m$ complex numbers $\lambda_{j}$ and state components $z_{\lambda_{j}}$ in $\mathbb{C}^{2}$.

\section{B. Solution $T_{\lambda}$ of the partial differential equation}

Following section II, to design the function $T^{*}$, we first look for a function $T_{\lambda}: \mathbb{R}^{4} \times \mathbb{R}^{2} \times \mathbb{R} \rightarrow \mathbb{C}^{2}$ solution of :

$\lambda T_{\lambda}(\mathcal{X}, \psi, t)+\psi=\frac{\partial T_{\lambda}}{\partial \mathcal{X}}(\mathcal{X}, \psi, t)[\mathcal{A}(t) \mathcal{X}+\mathcal{B}(t)]$

\footnotetext{
${ }^{1}$ An alternative to (8) is obtained by replacing $\frac{R_{s}}{L} \psi_{i}$ by $\frac{R_{s}}{L} y_{i}$ :$$
\dot{\psi}_{1}=-\mathcal{X}_{3}\left(\frac{J}{L P} \mathcal{X}_{2}+y_{2}\right)+\left(\frac{u_{1}}{L}-\frac{R_{s}}{L} y_{1}\right)
$$$$
\dot{\psi}_{2}=-\mathcal{X}_{3}\left(\frac{J}{L P} \mathcal{X}_{1}-y_{1}\right)+\left(\frac{u_{2}}{L}-\frac{R_{s}}{L} y_{2}\right)
$$$$
{ }^{2} \text { Again, here } \psi_{j} \text { can be replaced by } y_{j} \text {. }
$$ 


$$
\begin{aligned}
& +\frac{\partial T_{\lambda}}{\partial \psi}(\mathcal{X}, \psi, t)\left[\mathcal{C}(t) \psi+\sum_{i=1}^{2} e_{i}\left(\mathcal{X}^{T} \mathcal{D}_{i} \mathcal{X}\right)+\mathcal{E}(t) \mathcal{X} \mathcal{F}(t)\right] \\
& +\frac{\partial T_{\lambda}}{\partial t}(\mathcal{X}, \psi, t)
\end{aligned}
$$

From (2) and (10), a solution is given by :

$$
\begin{aligned}
T_{\lambda}(\mathcal{X}, \psi, t)=\int_{-\infty}^{t} \exp (\lambda(t-s))\left[\Phi_{\psi}(s, t) \psi\right. \\
+\sum_{i=1}^{2} e_{i}\left(\mathcal{X}^{T} \Psi_{\psi \mathcal{X}, 2 i}(s, t) \mathcal{X}\right) \\
\left.+\Psi_{\psi \mathcal{X}, 1}(s, t) \mathcal{X}+\Psi_{\psi \mathcal{X}, 0}(s, t)\right] d s
\end{aligned}
$$

Hence $T_{\lambda}$ is a polynomial of the second degree in $\mathcal{X}$ and first degree in $\psi$, i.e. ${ }^{3}$ :

$$
\begin{aligned}
T_{\lambda}(\mathcal{X}, \psi, t)=P_{\lambda}(t) \psi+\sum_{i=1}^{2} & e_{i}\left(\mathcal{X}^{T} Q_{\lambda, i}(t) \mathcal{X}\right) \\
& +R_{\lambda}(t) \mathcal{X}+S_{\lambda}(t)
\end{aligned}
$$

To find $\left(P_{\lambda}, Q_{\lambda, i}, R_{\lambda}, S_{\lambda}\right)$, we do not evaluate the above integral off-line since. Instead, we substitute this expression of $T_{\lambda}$ in (13). This yields :

$$
\begin{aligned}
\dot{P}_{\lambda}= & \lambda P_{\lambda}-P_{\lambda} \mathcal{C}(t)+I \\
\dot{Q}_{\lambda, i}= & \lambda Q_{\lambda, i}-\left[Q_{\lambda, i} \mathcal{A}(t)+\mathcal{A}(t)^{T} Q_{\lambda, i}\right] \\
& -\sum_{j=1}^{2}\left(e_{i}^{T} P_{\lambda} e_{j}\right) \mathcal{D}_{j} \\
\dot{R}_{\lambda}= & \lambda R_{\lambda}-2 \sum_{i=1}^{2} e_{i} \mathcal{B}(t)^{T} Q_{\lambda, i} \\
& -P_{\lambda} \mathcal{E}(t)-R_{\lambda} \mathcal{A}(t) \\
\dot{S}_{\lambda}= & \lambda S_{\lambda}-P_{\lambda} \mathcal{F}(t)-R_{\lambda} \mathcal{B}(t)
\end{aligned}
$$

If the input/output signals (4) are bounded functions of time, so are the functions $\mathcal{A}$ to $\mathcal{F}$. Then, because of the lower triangular structure of (15), if the real part of $\lambda$ is chosen strictly smaller than $-\sup _{t}\{|\mathcal{C}(t)|, 2|\mathcal{A}(t)|\}$, then each solution $t \mapsto\left(P_{\lambda}(t), Q_{\lambda, i}(t), R_{\lambda}(t), S_{\lambda}(t)\right)$ of (15) is bounded whatever its initial condition is.

\section{Injectivity of $T$}

We follow [2, Section 2.4] to study the injectivity of the function $T=\left(T_{\lambda_{1}}, \ldots, T_{\lambda_{m}}\right)$ First we approximate the solution $t \mapsto\left(P_{\lambda}(t), Q_{\lambda, i}(t), R_{\lambda}(t), S_{\lambda}(t)\right)$ for $|\lambda|$ large. Assuming that the input/output signals (4) and their first two derivatives are bounded functions of time, we can find a positive real number $\ell$, such that, given the initial condition $\left(P_{\lambda}(0), Q_{\lambda, i}(0), R_{\lambda}(0), S_{\lambda}(0)\right)$, there exist real numbers $M_{1}$ and $M_{2}$, such that, for each $\lambda$ in $\mathbb{C}$ with real part smaller than $-\ell$ and all $t \geq 0$, we have ${ }^{4}$ :

\footnotetext{
${ }^{3} Q_{\lambda, i}$ is a matrix with symmetric real and imaginary parts, $P_{\lambda}$ and $R_{\lambda}$ are matrices and $S_{\lambda}$ is a vector.

${ }^{4}$ Observe that each term between the bars multiplied by $\lambda^{4}$ is a solution of a differential equation and as such is bounded in both $t$ and $\lambda$ (hence $M_{2}$ ) provided their initial condition is appropriately chosen (hence $M_{1}$ ).
}

$$
\begin{aligned}
& M_{1} \exp \left(\frac{\operatorname{Real}(\lambda)}{2} t\right)+\frac{M_{2}}{|\lambda|^{4}} \geq \\
& \left|P_{\lambda}(t)+\frac{I}{\lambda}+\frac{\mathcal{C}(t)}{\lambda^{2}}+\frac{\mathcal{\mathcal { C }}(t)+\mathcal{C}^{2}}{\lambda^{3}}\right| \\
& +\sum_{i=1}^{2}\left|Q_{\lambda, i}(t)+\frac{\mathcal{D}}{\lambda^{2}}-\frac{\mathcal{D}_{i} \mathcal{A}(t)+\mathcal{A}(t)^{T} \mathcal{D}_{i}+\sum_{j=1}^{2}\left(e_{i}^{T} C(t) e_{j}\right) \mathcal{D}_{j}}{\lambda^{3}}\right| \\
& +\left|R_{\lambda}(t)+\frac{\mathcal{E}(t)}{\lambda^{2}}+\frac{\dot{\mathcal{E}}(t)+\mathcal{C}(t) \mathcal{E}(t)+\mathcal{E}(t) \mathcal{A}(t)+2 \sum_{j=1}^{2} e_{i} \mathcal{B}(t)^{T} \mathcal{D}_{i}}{\lambda^{3}}\right| \\
& +\left|S_{\lambda}(t)+\frac{\mathcal{F}(t)}{\lambda^{2}}+\frac{\dot{\mathcal{F}}(t)+\mathcal{C}(t) \mathcal{F}(t)+\mathcal{E}(t) \mathcal{B}(t)}{\lambda^{3}}\right| .
\end{aligned}
$$

The expansion in $\frac{1}{\lambda}$, stopped here to order 3 to avoid messy expressions, can be done to any order $p$ provided the input/output signals and their $p-1$ first derivatives are bounded. Actually to proceed, we need to go up to order $p=4$.

Let $\widehat{T}_{\lambda}$ be the approximation of $T_{\lambda}$ we obtain with an expansion up to order 4 . It is :

$$
\begin{aligned}
& \widehat{T}_{\lambda}(\mathcal{X}, \psi, t)=\frac{\mathfrak{T}_{0}(\mathcal{X}, \psi, t)}{\lambda}+\frac{\mathfrak{T}_{1}(\mathcal{X}, \psi, t)}{\lambda^{2}} \\
& +\frac{\mathfrak{T}_{2}(\mathcal{X}, \psi, t)}{\lambda^{3}}+\frac{\mathfrak{T}_{3}(\mathcal{X}, \psi, t)}{\lambda^{4}}
\end{aligned}
$$$$
\text { with } \mathfrak{T}_{j} \text { given by : }
$$

$$
\begin{aligned}
\mathfrak{T}_{0}(\mathcal{X}, \psi, t)= & \psi \\
\mathfrak{T}_{1}(\mathcal{X}, \psi, t)= & \mathcal{C}(t) \psi+\mathcal{X}^{T} \mathcal{D} \mathcal{X}+\mathcal{E}(t) \mathcal{X}+\mathcal{F}(t) \\
\mathfrak{T}_{2}(\mathcal{X}, \psi, t)= & {[\dot{\mathcal{C}}(t) \psi+\dot{\mathcal{E}}(t) \mathcal{X}+\dot{\mathcal{F}}(t)] } \\
& \quad+\mathcal{C}(t)\left[\mathcal{C}(t) \psi+\mathcal{X}^{T} \mathcal{D} \mathcal{X}+\mathcal{E}(t) \mathcal{X}+\mathcal{F}(t)\right] \\
& +\left[2 \mathcal{X}^{T} \mathcal{D}+\mathcal{E}(t)\right][\mathcal{A}(t) \mathcal{X}+\mathcal{B}(t)] \\
\mathfrak{T}_{3}(\mathcal{X}, \psi, t)= & \ldots
\end{aligned}
$$

The key point here is that $\mathfrak{T}_{j}(\mathcal{X}, \psi, t)$ is the $j$ th derivative of the output $y=\psi$ for a solution at the point $(\mathcal{X}, \psi)$ at time $t$. Specifically, with (6) and (9), let $\mathfrak{L}$ be the following differential operator, acting on functions of $(\mathcal{X}, \psi, t)$,

$$
\begin{array}{r}
\mathfrak{L} \bullet(\mathcal{X}, \psi, t)=\frac{\partial \bullet}{\partial \mathcal{X}}(\mathcal{X}, \psi, t)[\mathcal{A}(t) \mathcal{X}+\mathcal{B}(t)]+\frac{\partial \bullet}{\partial t}(\mathcal{X}, \psi, t) \\
+\frac{\partial \bullet}{\partial \psi}(\mathcal{X}, \psi, t)\left[\mathcal{C}(t) \psi+\mathcal{X}^{T} \mathcal{D} \mathcal{X}+\mathcal{E}(t) \mathcal{X}+\mathcal{F}(t)\right]
\end{array}
$$

We have :

$$
\mathfrak{T}_{j}=\mathfrak{L}^{j} \psi .
$$

Now, with (16) (up to order 4), given a compact subset of $\mathfrak{S}$, there exist $N_{1}$ and $N_{2}$ such that, for any two pairs $\left(\mathcal{X}_{a}, \psi_{a}\right)$ and $\left(\mathcal{X}_{b}, \psi_{b}\right)$ in this set, we have

$$
\begin{aligned}
\left|\widehat{T}_{\lambda}\left(\mathcal{X}_{a}, \psi_{a}, t\right)-\widehat{T}_{\lambda}\left(\mathcal{X}_{b}, \psi_{b}, t\right)\right| \\
\leq \quad\left|T_{\lambda}\left(\mathcal{X}_{a}, \psi_{a}, t\right)-T_{\lambda}\left(\mathcal{X}_{b}, \psi_{b}, t\right)\right| \\
\quad+\left|\left(\mathcal{X}_{a}, \psi_{a}\right)-\left(\mathcal{X}_{b}, \psi_{b}\right)\right|\left[N_{1} \exp \left(\frac{\operatorname{Real}(\lambda)}{2} t\right)+\frac{N_{2}}{|\lambda|^{5}}\right] \\
\leq \quad\left|\sum_{j=1}^{4} \frac{\mathfrak{\mathcal { L }}^{j-1} \psi\left(\mathcal{X}_{a}, \psi_{a}, t\right)-\mathfrak{L}^{j-1} \psi\left(\mathcal{X}_{b}, \psi_{b}, t\right)}{\lambda^{j}}\right| \\
\quad+\left|\left(\mathcal{X}_{a}, \psi_{a}\right)-\left(\mathcal{X}_{b}, \psi_{b}\right)\right|\left[N_{1} \exp \left(\frac{\operatorname{Real}(\lambda)}{2} t\right)+\frac{N_{2}}{\left.\lambda\right|^{5}}\right]
\end{aligned}
$$


From studying the observability of (3), it can be derived that, given $\varepsilon>0$, there exists $K$ such that, for each $t$ for which the angular velocity satisfies $|\omega(t)|=$ $\left|\mathcal{X}_{3}(t)\right| \geq \varepsilon$, and for any two pairs $\left(\mathcal{X}_{a}, \psi_{a}\right)$ and $\left(\mathcal{X}_{b}, \psi_{b}\right)$ in the given compact set, we have :

$$
\begin{aligned}
& \left|\left(\mathcal{X}_{a}, \psi_{a}\right)-\left(\mathcal{X}_{b}, \psi_{b}\right)\right| \leq \\
& K \mid\left(\psi_{a}, \mathfrak{L} \psi\left(\mathcal{X}_{a}, \psi_{a}\right), \mathfrak{L}^{2} \psi\left(\mathcal{X}_{a}, \psi_{a}\right), \mathfrak{L}^{3} \psi\left(\mathcal{X}_{a}, \psi_{a}\right)\right) \\
& \quad-\left(\psi_{b}, \mathfrak{L} \psi\left(\mathcal{X}_{b}, \psi_{b}\right), \mathfrak{L}^{2} \psi\left(\mathcal{X}_{b}, \psi_{b}\right), \mathfrak{L}^{3} \psi\left(\mathcal{X}_{b}, \psi_{b}\right)\right) \mid
\end{aligned}
$$

So by :

- selecting the number $m$ larger or equal to 4 ,

- choosing $m$ distinct complex numbers $\lambda_{j}$ with real part smaller than $-\ell$ and sufficiently large in modulus to verify $\frac{N_{2}}{\left|\lambda_{j}\right|^{5}} \leq \frac{1}{3}$,

the function $(\mathcal{X}, \psi) \in \mathfrak{S} \mapsto T(\mathcal{X}, \psi, t)=$ $\left(\ldots T_{\lambda_{j}}(\mathcal{X}, \psi, t) \ldots\right)$ is injective, for each sufficiently large $t$ verifying $N_{1} \exp \left(\frac{\max _{j}\left\{\operatorname{Real}\left(\lambda_{j}\right)\right\}}{2} t\right) \leq \frac{1}{3}$ and such that $|\omega(t)|=\left|\mathcal{X}_{3}(t)\right| \geq \varepsilon$.

\section{Expression of $T^{*}$}

Instead of writing an expression for $T^{*}$, we propose an algorithm to compute it. It is based on the minimization in $(\mathcal{X}, \psi)$ at each time $t$, of the criterion :

$$
\begin{array}{r}
\mathfrak{J}(\mathcal{X}, \psi, t)=\sum_{j=1}^{m} p_{j}\left|z_{\lambda_{j}}-T_{\lambda_{j}}(\mathcal{X}, \psi, t)\right|^{2} \\
+p_{0}\left[\left(\mathcal{X}_{1}-\frac{L P}{J} \psi_{1}\right)^{2}+\left(\mathcal{X}_{2}+\frac{L P}{J} \psi_{2}\right)^{2}-\frac{P^{2} \Phi^{2}}{J^{2}}\right]^{2}
\end{array}
$$

where the $p_{j}$ are weights. Minimizing this criterion says that we solve the equation :

$$
\frac{\partial \mathfrak{J}}{\partial(\mathcal{X}, \psi)}(\mathcal{X}, \psi, t)=0
$$

But now, if $\left(\hat{\mathcal{X}}_{*}(t), \hat{\psi}_{*}(t)\right)$ is a solution of (18) at each time $t$, assumed to be differentiable in $t$, we have also :

$\frac{\partial^{2} \mathfrak{\jmath}}{\partial(\mathcal{X}, \psi)^{2}}(\mathcal{X}, \psi, t)\left(\begin{array}{c}\dot{\hat{\mathcal{X}}}_{*}(t) \\ \hat{\hat{\psi}}_{*}(t)\end{array}\right)+\frac{\partial^{2} \mathfrak{\jmath}}{\partial t \partial(\mathcal{X}, \psi)}\left(\hat{\mathcal{X}}_{*}(t), \hat{\psi}_{*}(t), t\right)=0$

So, if the Hessian $\frac{\partial^{2} \mathfrak{J}}{\partial(\mathcal{X}, \psi)^{2}}$ is invertible, $\left(\hat{\mathcal{X}}_{*}(t), \hat{\psi}_{*}(t)\right)$ is a particular solution of the system :

$\left(\begin{array}{c}\dot{\hat{\mathcal{X}}} \\ \hat{\hat{\psi}}\end{array}\right)=-\left(\frac{\partial^{2} \mathfrak{J}}{\partial(\mathcal{X}, \psi))^{2}}(\hat{\mathcal{X}}, \hat{\psi}, t)\right)^{-1} \frac{\partial^{2} \mathfrak{\jmath}}{\partial t \partial(\mathcal{X}, \psi)}(\hat{\mathcal{X}}, \hat{\psi}, t)$

and, in view of (18), also of :

$$
\begin{array}{r}
\left(\begin{array}{c}
\dot{\hat{\mathcal{X}}} \\
\dot{\hat{\psi}}
\end{array}\right)=-\left(\frac{\partial^{2} \mathfrak{J}}{\partial(\mathcal{X}, \psi)^{2}}(\hat{\mathcal{X}}, \hat{\psi}, t)\right)^{-1} \frac{\partial^{2} \mathfrak{J}}{\partial t \partial(\mathcal{X}, \psi)}(\hat{\mathcal{X}}, \hat{\psi}, t) \\
-M(\hat{\mathcal{X}}, \hat{\psi}, t) \frac{\partial \mathfrak{\mathcal { J }}}{\partial(\mathcal{X}, \psi)}(\hat{\mathcal{X}}, \hat{\psi}, t)
\end{array}
$$

So the idea to get the estimation $(\hat{\mathcal{\chi}}, \hat{\psi})$ from the $z_{\lambda_{j}}$ is to solve online this system (19) starting from an $a d-$ hoc initial condition and choosing the matrix $M$ above positive definite in order to make the set defined by the equation (18) attractive. This initial condition should be solution at time $t=0$ of (18). A procedure for the initialization is as follows : choose initial conditions $\left(P_{\lambda_{j}}(0), Q_{\lambda_{j}}(0), R_{\lambda_{j}}(0), S_{\lambda_{j}}(0)\right.$ and $(\hat{\mathcal{\chi}}(0), \hat{\psi}(0))$ as close as possible to the physical data, then define the initial condition $z_{\lambda_{j}}(0)$ simply as :

$$
z_{\lambda_{j}}(0)=T_{\lambda_{j}}(\hat{\mathcal{X}}(0), \hat{\psi}(0), 0) .
$$

In this process of solving online the system (19), the only problem we may encounter is a singularity of the Hessian $\frac{\partial^{2} \mathfrak{J}}{\partial(\mathcal{X}, \psi)^{2}}$. The existence of singularity points is related either to the one of local minima in $(\mathcal{X}, \psi)$ of the criterion $\mathfrak{J}$ or to a degeneracy of the global minimum Finally, we note that this process is a particular case of the celebrated Davidenko's continuation method with a stabilization term (see [1, Section 2]). However, a specificity here is that the path and its parametrization are imposed.

We end this subsection by observing that another way for minimizing $\mathfrak{J}$ follows from replacing, in its expression, the products $\mathcal{X}_{1} \mathcal{X}_{i}$ and $\mathcal{X}_{2} \mathcal{X}_{i}$, which appear in $\sum_{i=1}^{2} e_{i}\left(\mathcal{X}^{T} \mathcal{D}_{i} \mathcal{X}\right)$ involved in $T_{\lambda_{j}}$ in (9), by independent variables, say $\pi_{1 i}$ and $\pi_{2 i}$. This makes $\mathfrak{J}$ a polynomial of the second degree in $(\mathcal{X}, \psi, \pi)$ and allows its minimization with respect to these variables by a matrix inversion only. The preliminary simulations we have show that it is an efficient method giving good results as long as the angular velocity is not too small.

\section{OBSERVER EQUATIONS}

To recap, the observer we propose is defined by choosing $m \geq 4$ complex numbers $\lambda_{j}, m+1$ weights $p_{i}>0$ and stiffness coefficients $k_{\mathcal{X}_{j}}$ and $k_{\psi_{j}}$. The observer is then given by the dynamical system made of (12) and (15), with $\lambda=\lambda_{j}$ for $j \in\{1, \ldots, m\}$, (19) with :

$$
M(\hat{\mathcal{X}}, \hat{\psi}, t)=\operatorname{diag}\left(k_{\mathcal{X}_{1}}, k_{\mathcal{X}_{2}}, k_{\mathcal{X}_{3}}, k_{\mathcal{X}_{4}}, k_{\psi_{1}}, k_{\psi_{2}}\right)
$$

and $\mathfrak{J}$ given by (17) where $T_{\lambda_{j}}$ is given by (14). It gives the estimates :

$$
\hat{\theta}=2 \arctan \left(\frac{b}{a+\sqrt{a^{2}+b^{2}}}\right), \hat{\omega}=\hat{\mathcal{X}}_{3}, \hat{\tau}_{l}=J \hat{\mathcal{X}}_{4},
$$

where we have defined ${ }^{5}$ :

$$
a:=J \hat{\mathcal{X}}_{2}+L P i_{\beta}(t), b:=J \hat{\mathcal{X}}_{1}-L P i_{\alpha}(t) .
$$

\section{Simulation RESUlts}

In this section we present simulation results to show the performance of the proposed observer. The input-output signals used by the observer are generated from the following two-phase dq (Blondel-Park) model expressed in a frame rotating at speed $\omega$ and with DC and second harmonic (saliency effect) for the inductance and first harmonic for the flux,

\footnotetext{
${ }^{5}$ We can replace $i_{\alpha}(t)$ and $i_{\beta}(t)$ respectively by $\hat{\psi}_{1}$ and $\hat{\psi}_{2}$.
} 

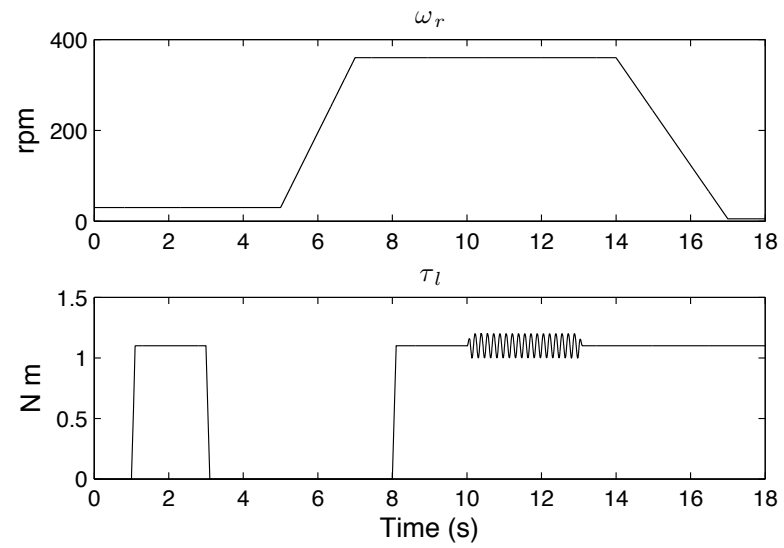

Fig. 1. Reference angular velocity and load torque

$$
\begin{aligned}
L_{d} \stackrel{\dot{i_{d}}}{\dot{d_{d}}} & =-R_{s} i_{d}+\omega L_{q} i_{q}+v_{d} \\
L_{q} \hat{i}_{q} & =-R_{s} i_{q}-\omega L_{d} i_{d}-\Phi \omega+v_{q} \\
J \dot{\omega} & =P\left[\left(L_{q}-L_{d}\right) i_{d} i_{q}+\Phi i_{q}\right]-B \omega-\tau_{l} \\
\dot{\theta} & =\omega \\
\left(\begin{array}{cc}
i_{\beta} & v_{\beta} \\
i_{\alpha} & v_{\alpha}
\end{array}\right) & =\left(\begin{array}{cc}
\cos (\theta) & \sin (\theta) \\
-\sin (\theta) & \cos (\theta)
\end{array}\right)\left(\begin{array}{ll}
i_{q} & v_{q} \\
i_{d} & v_{d}
\end{array}\right)
\end{aligned}
$$

The model (3) is recovered when $L_{d}=L_{q}=L$.

We test the observer for a motor with the following characteristics :

$$
\begin{gathered}
P=2, \Phi=0.1994 \mathrm{Web} ., \\
L_{d}=0.0061 \mathrm{mH}, L_{q}=0.0121 \mathrm{mH}, R_{s}=1.45 \Omega, \\
J=0.0011 \mathrm{~kg} \cdot \mathrm{m}^{2}, B=0.0009 \mathrm{~kg} \cdot \mathrm{m}^{2} / \mathrm{s} .
\end{gathered}
$$

We simulate the motor in closed-loop with a simple cascaded-loop linear controller. The motor is supposed to be equipped with an incremental angular encoder. From this angular position measurement, the estimated angular velocity is obtained via a dirty derivative. This allows us to control the model $(20)$ via $\left(v_{d}, v_{q}\right)$ obtained by :

- A PI controller on the angular velocity to regulate it at its desired value, the integrator being needed to take care of the unknown torque. This gives us reference signals for $\left(i_{d}, i_{q}\right)$.

- A P controller on $\left(i_{\alpha}, i_{\beta}\right)$ to regulate this pair to the above defined reference pair.

The speed reference $\omega_{r}$ and load torque $\tau_{l}$ profiles used for the test are depicted. on Figure 1. We end with a very small desired angular velocity to study the observer in a region close to non-injectivity of $T$. Also we have a periodic disturbing torque (of eccentricity type) between $12 s$ and $15 s$ to test the behavior robustness when the assumption $\dot{\mathcal{X}}_{4}=0$ is violated.

The input-output signals for the observer (see (4)) which we obtain from this simulation are depicted on Figure 2. They are collected at a sampling rate of $1 \mathrm{~ms}$. Then the sampled version of $i_{\alpha}, i_{\beta}, v_{\alpha}$ and $v_{\beta}$ are corrupted by a colored noise $\left(\frac{1}{s+75}\right)$ with amplitude equal
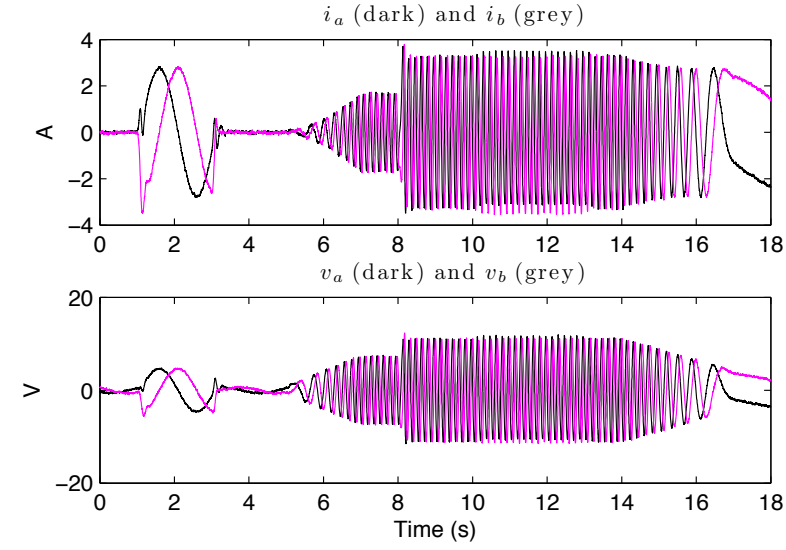

Fig. 2. Observer input-output signals

to $1 \%$ of the respective signals.

The observer design is made from the model (5) and (7) but in the latter the $\psi_{j}$ are replaced by the "measurements" $y_{j}$ with the consequence that the matrix $\mathcal{C}$ in (9) is zero. We have picked $m=5 \lambda_{i}$ as :

$$
\begin{gathered}
-25.0+25.0 i,-37.5+37.5 i,-50.0+50.0 i, \\
-62.5+62.5,-75.0+75.0 i .
\end{gathered}
$$

The criterion weights $p_{j}$ are all equal to 1 , except $p_{0}=2$. The matrix $M$ in (19) is chosen as:

$$
M=\operatorname{diag}(450,450,1125,225,450,450) \text {. }
$$

The differential equations involved in the observer implementation of the are solved with an elementary explicit Euler scheme with time step equal to the sampling rate, i.e., $1 \mathrm{~ms}$.

When the parameters used by the observer are the same as the ones used for the motor, except for $L$ and $B$, i.e.

$$
\begin{gathered}
P=2, \Phi=0.1994 \text { Web } . \\
L=0.0091 \mathrm{mH}, R_{s}=1.45 \Omega \\
J=0.0011 \mathrm{~kg} \cdot \mathrm{m}^{2}, B=0 \mathrm{~kg} \cdot \mathrm{m}^{2} / \mathrm{s}
\end{gathered}
$$

we get the estimates depicted on Figure 3. Although we have noisy input-output signals and saliency effects we get a very satisfactory response except, as predicted, when the angular velocity becomes too small as can be seen at the end of the simulation.

When the parameters used by the observer differ significantly from the ones used for the motor (i.e., $50 \%$ for $R_{s}, 15 \%$ for $\Phi$ and $20 \%$ for $L$ ) we get Figure 4 for the set of parameters

$$
\begin{gathered}
P=2, \Phi=0.2293 \mathrm{Web} . \\
L=0.0109 \mathrm{mH}, R_{s}=2.1750 \Omega \\
J=0.0011 \mathrm{~kg} \cdot \mathrm{m}^{2}, B=0 \mathrm{~kg} \cdot \mathrm{m}^{2} / \mathrm{s} \\
\text { gure } 5 \text { for } \\
\quad P=2, \Phi=0.1695 \mathrm{Web} . \\
L=0.0073 \mathrm{~m} H, R_{s}=0.7250 \Omega \\
J=0.0011 \mathrm{~kg} \cdot \mathrm{m}^{2}, B=0.0000 \mathrm{~kg} \cdot \mathrm{m}^{2} / \mathrm{s}
\end{gathered}
$$

Figure 5 for

The parameters $m, \lambda_{j}, p_{i}, k_{\mathcal{X}_{j}}$ and $k_{\psi_{j}}$ have hardly been tuned in these simulations. Thus, although the results are satisfactory, they should be considered as 

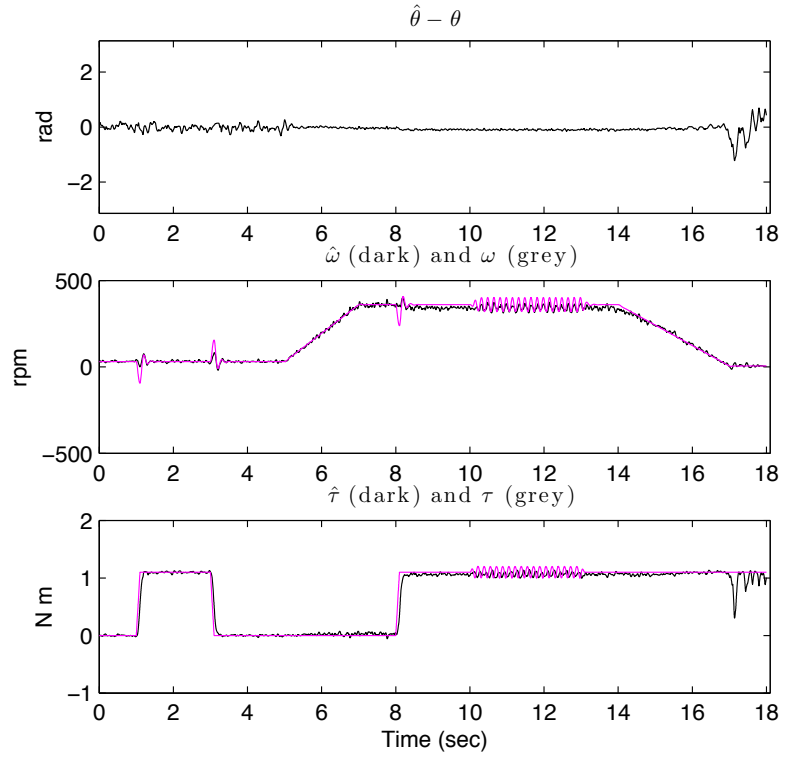

Fig. 3. Observer results for the parameter set (21)
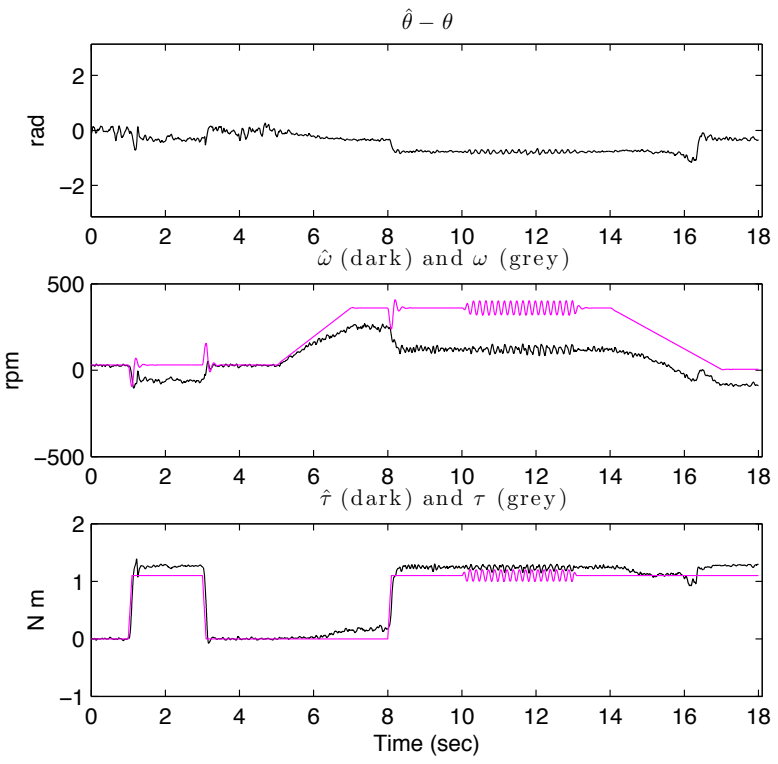

Fig. 4. Observer results for the parameter set (22)

preliminary.

\section{CONCLUDING REMARKS}

We have presented a study of feasibility of application of KKL observers to the problem of estimation of mechanical coordinates in electrical machines. Several issues need to be clarified before this procedure can be considered as a sensible practical alternative to the existing techniques :

- Assessment of the impact on the performance of the observer of its tuning parameters, i.e., $m, \lambda_{j}, M$ and $p_{j}$, and the development of a systematic procedure for
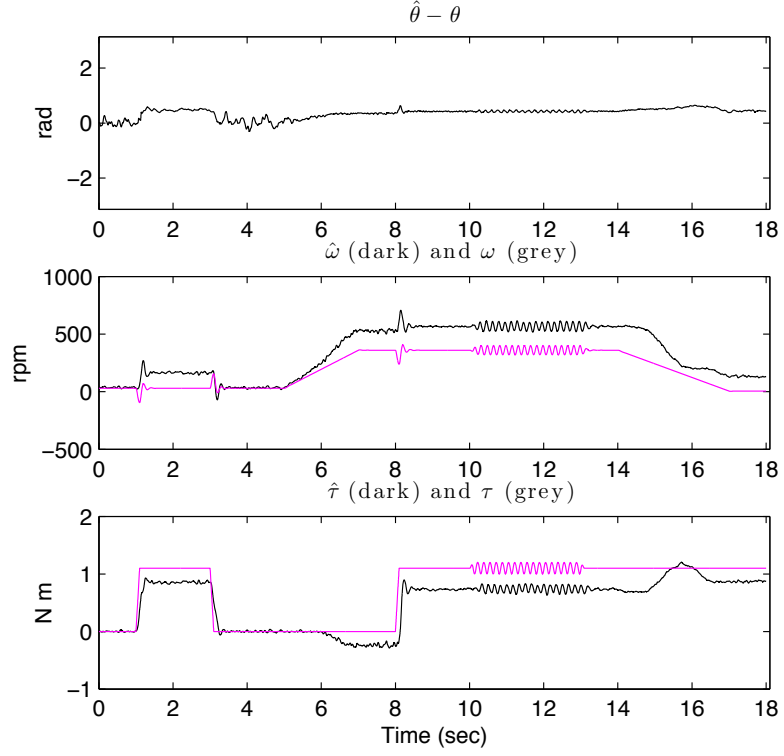

Fig. 5. Observer results for the parameter set (23)

their selection should be further investigated. Given that there is a lot of flexibility in the design of KKL observers, there is hope for significant improvement in specific applications, like the one at hand.

- The procedure proposed here, that involves a minimum search, is computationally too demanding for on-line implementation. A simplified version of the observer, that retains its essential features, is currently being studied.

\section{REFERENCES}

[1] E. L. Allgower, K. Georg, Simplicial and continuation methods for approximating, fixed points and solutions to systems of equations. SIAM Review, Volume 22, 28-85, 1980.

[2] V. Andrieu, L. Praly, On the existence of a Kazantzis-Kravaris / Luenberger observer. SIAM J. Control Optim. Vol. 45, No. 2, pp 432-456, 2006.

[3] G. Besançon (Ed.), Nonlinear Observers and Applications. Lecture Notes in Control and Information Science 363, Springer Verlag, 2007.

[4] S. Ibarra, J. Moreno and G. Espinosa-Perez, Global observability analysis of sensorless induction motors. Automatica, Vol 40, pp. 1079-1085, 2004.

[5] J. Chiasson, Modeling and high-performance control of electric drives. Wiley 2005.

[6] N. Kazantzis, C. Kravaris, Nonlinear observer design using Lyapunov's auxiliary theorem. Systems \& Control Letters, 34 (1998), pp. 241-247.

[7] Krause P.C., Analysis of Electric Machinery. McGraw Hill, New York 1986

[8] D. Luenberger, Observing the state of a linear system, IEEE Trans. Military Electronics, MIL-8, (1964), pp. 74-80.

[9] R. Marino, P. Tomei and P. Verelli, A nonlinear tracking control for sensorless induction motors, Automatica, Vol 42, pp. 1637$1650,2006$.

[10] M. Montanari, S. Peresada and A. Tilli, A speed sensorless indirect field oriented control for induction motors based on high-gain speed estimation, Automatica, Vol 42, pp. 1637-1650, 2006.

[11] P. Vas, Sensorless Vector and Direct Torque Control, Oxford Press, 1998. 